\title{
Stabilization of partially folded states in protein folding/misfolding transitions by hydrostatic pressure
}

S.T. Ferreira ${ }^{1}$, A. Chapeaurouge ${ }^{2}$ and F.G. De Felice ${ }^{1}$

\author{
${ }^{1}$ Programa de Bioquímica e Biofísica Celular, Instituto de Bioquímica Médica, \\ Universidade Federal do Rio de Janeiro, Rio de Janeiro, RJ, Brasil \\ ${ }^{2}$ Departamento de Fisiologia e Farmacodinâmica, Instituto Oswaldo Cruz, \\ Fiocruz, Rio de Janeiro, RJ, Brasil
}

\author{
Correspondence \\ S.T. Ferreira \\ Programa de Bioquímica e \\ Biofísica Celular \\ Instituto de Bioquímica Médica \\ UFRJ \\ 21944-590 Rio de Janeiro, RJ \\ Brasil \\ Fax: +55-21-2562-6789 \\ E-mail: ferreira@bioqmed.ufrj.br \\ Presented at the 3rd International \\ Conference on High Pressure \\ Bioscience and Biotechnology, \\ Rio de Janeiro, RJ, Brazil, \\ September 27-30, 2004. \\ Research in the laboratory of \\ S.T. Ferreira was supported by \\ grants from the Howard Hughes \\ Medical Institute, FAPERJ and CNPq.
}

Received January 24, 2005 Accepted April 12, 2005

\begin{abstract}
In the last few years, hydrostatic pressure has been extensively used in the study of both protein folding and misfolding/aggregation. Compared to other chemical or physical denaturing agents, a unique feature of pressure is its ability to induce subtle changes in protein conformation, which allow the stabilization of partially folded intermediate states that are usually not significantly populated under more drastic conditions (e.g., in the presence of chemical denaturants or at high temperatures). Much of the recent research in the field of protein folding has focused on the characterization of folding intermediates since these species appear to be involved in a variety of diseasecausing protein misfolding and aggregation events. The exact mechanisms of these biological phenomena, however, are still poorly understood. Here, we review recent examples of the use of hydrostatic pressure as a tool to obtain insight into the forces and energetics governing the productive folding or the misfolding and aggregation of proteins.
\end{abstract}

Key words

- High pressure

- Protein folding

- Misfolding

- Amyloid

- Aggregation

\section{Introduction}

Protein folding has evolved from a field of mere academic interest to an area of major biological and medical relevance. In part, the increasing interest is due to the recognition that failure of cellular protein folding mechanisms is associated with a variety of important human disorders ranging from cystic fibrosis to Alzheimer's disease. A growing body of evidence indicates a critical role for partially folded protein conformers in the process of conversion of normal cellular proteins into disease-causing, proteaseresistant protein aggregates of various mor- phologies $(1,2)$.

An elegant approach to the investigation of the mechanisms of protein folding is the use of de novo designed proteins (3). These small, artificial proteins represent simplified versions of their natural counterparts, yet they retain all the inherent features necessary for productive folding. The successful de novo design of a given protein fold provides insight into the contributions of different types of non-covalent interactions involved in the stabilization of its three-dimensional structure. A common target for the design of proteins from scratch has been the three-helix bundle folding motif, as it repre- 
sents one of the simplest tertiary structures known. Derived from sequence patterns encountered in natural coiled coil proteins, amphiphilic helical bundles stabilized by a hydrophobic core have been successfully designed $(4,5)$.

Whereas folding of small, single-domain polypeptides ( $<100$-amino acid residues) is generally thought to be a highly cooperative process, more complex folding pathways, including the presence of well-defined intermediate states, are usually considered to be characteristic of larger proteins. Recent kinetic simulation studies, however, have suggested a possible critical contribution of onpathway intermediates in the folding of a simple three-helix bundle motif (6). The fact that such intermediates are not usually detected in equilibrium or kinetic investigations of small, fast folding proteins suggests that they constitute metastable states that are very little populated under the conditions that are normally employed to study protein folding (typically involving the use of chemical denaturants, acidic $\mathrm{pH}$ or high temperatures).

According to Le Chatelier's principle, application of hydrostatic pressure shifts the equilibrium of any physical system towards the state that occupies a smaller volume (corresponding to a negative volume change). Various factors may contribute to the change in specific volume of proteins upon denaturation, including i) exposure of charged groups that become hydrated and cause solvent electrostriction ( $\Delta \mathrm{V} \sim-10 \mathrm{ml} / \mathrm{mol})$, ii) hydration of previously buried hydrophobic side chains, and iii) elimination of solventinaccessible void volumes and packing defects in the folded structure. Experimentally determined volume changes of protein unfolding fall in the range of -30 to $-300 \mathrm{ml} /$ mol, which usually accounts for about $0.5 \%$ of the total protein volume $(7,8)$. Most of the charged side chains in proteins are usually surface-exposed in their native states, since up to $19 \mathrm{kcal} / \mathrm{mol}$ are required for transfer of an unpaired charge from the aqueous solution to the low-dielectric environment of the protein core (9). Thus, contributions of electrostriction to the volume change upon protein denaturation are often considered to be relatively small. It should be noted, nevertheless, that the disruption of electrostatic interactions in the protein core, followed by the surface exposure of the two unpaired charges, may play a significant role in the volume change associated with unfolding. The importance of volume changes arising from the exposure of hydrophobic groups is still under debate. Based on the transfer of model compounds from non-polar solvents to water, it was initially concluded that the solvent-exposure of hydrophobic side chains is associated with a negative overall volume change (10). Other studies, however, have shown that interactions between aliphatic side chains and water result in positive volume changes (11). Pressure denaturation studies of both natural and de novo designed proteins in which the volumes occupied by internal cavities in the hydrophobic core were changed by amino acid substitutions indicate that the loss of internal void volumes plays a critical role in the unfolding of proteins induced by pressure $(12,13)$.

Pressure unfolding of proteins is determined by the change in volume and, therefore, leads to the stabilization of compact, more hydrated protein conformations, while the overall energy of the system remains constant. By contrast, varying the temperature changes both the volume and the thermal energy of the system, whereas addition of denaturing agents, such as guanidine hydrochloride or urea, requires consideration of the binding equilibria of those compounds to the protein. The use of pressure in investigations of the assembly of oligomeric proteins and larger aggregates has been extensively reviewed $(14,15)$. Therefore, here we will focus on pressure studies of the folding of single domain de novo designed and natural proteins, as well as on the effects of 
pressure on protein misfolding and aggregation phenomena.

\section{Pressure denaturation of de novo designed helical bundles}

We have recently synthesized a 65 -amino acid residue polypeptide (denoted $\alpha_{3-1}$ ) containing three helical regions joined by two (glycine) $)_{4}$ linkers (16) and examined its unfolding transitions induced by chemical denaturants $(\mathrm{GdnHCl})$, pressure and low temperature (17) in an attempt to stabilize possible folding intermediates. While $\mathrm{GdnHCl}$ induced (un)folding of $\alpha_{3-1}$ revealed a reversible, highly cooperative two-state transition between the native-like and the completely unfolded states, elevated pressures stabilized a metastable partially folded state of $\alpha_{3-1}$ (17). Another advantage of utilizing pressure as a thermodynamic variable is that the freezing point of water is significantly decreased at elevated pressures (18), so that cold denaturation experiments can be carried out without cryosolvent additives. The driving force of cold denaturation is the negative Gibbs free-energy change of solvation of the hydrophobic side chains at low temperature (19). The native state of proteins is mainly stabilized by interactions of non-polar groups, the hydration of which at low temperatures results in protein unfolding. The combined use of high pressures and low temperatures allowed the stabilization of yet another partially folded state of $\alpha_{3-1}$, as determined by intrinsic and bis-ANS fluorescence analysis. Using this approach, we were able to separate contributions from primarily hydrophilic and hydrophobic interactions involved in the different steps of folding of $\alpha_{3-1}$ (Figure 1).

In a subsequent study, three different $d e$ novo designed three-helix bundle proteins, designated GM-0, GM-1 and GM-2 and similar in their amino acid sequences to $\alpha_{3-1}$, were synthesized (13). While GM-0 was characterized by a native-like hydrophobic core similar to that of $\alpha_{3-1}$, the "mutants" GM-1 and GM-2 contained cavities created by either single or double replacements of large aliphatic side chains by smaller alanine residues, in an attempt to explore the folding tolerance of the helical bundle motif towards amino acid variability in its inner core. These proteins were independently unfolded by $\mathrm{GdnHCl}$, pressure or low temperatures (at $3.5 \mathrm{kbar}$ ) and revealed a remarkable folding plasticity: similar to the results obtained with $\alpha_{3-1}$, two molten-globule-like conformational intermediates could be detected in the unfolding transitions of GM-0 induced by high pressures and low temperatures. On the other hand, the cavity "mutants" GM-1 and GM-2 exhibited only one metastable state (stabilized at high pressures) that was different from both the native-like and fully unfolded states. These results point to the importance of hydrophobic core packing as a determinant not only of structure and stability, but also of the folding energetics of the threehelix bundle motif (13).

Denaturation studies of a de novo designed dimeric four-helix bundle protein (denoted $\left.\left(\alpha_{2}\right)_{2}\right)$ also revealed non-cooperative folding behavior for this protein (20). Spectroscopic and thermodynamic analysis showed that qualitatively similar monomeric

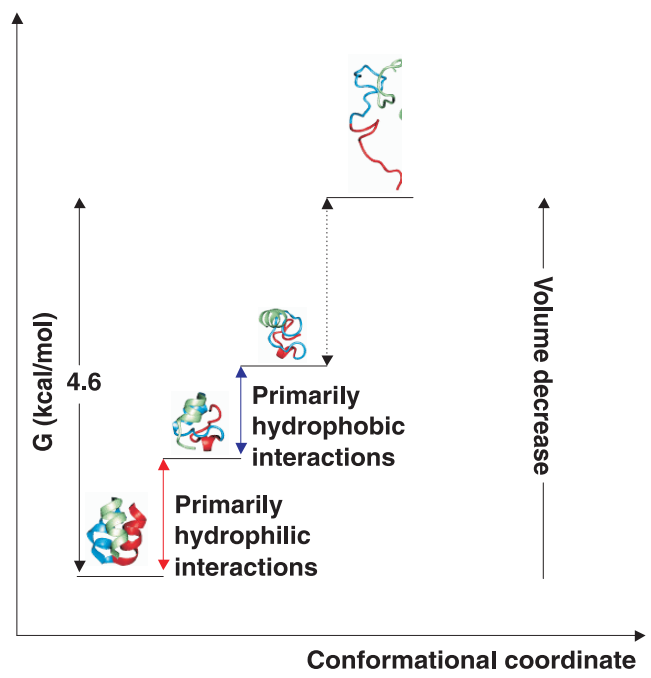

Figure 1. Thermodynamic scheme of the partial unfolding transitions of a de novo designed three-helix bundle protein, $\alpha_{3-1}$, induced by hydrostatic pressure (red arrow) and low temperature (at $3.5 \mathrm{kbar}$ of pressure, blue arrow). The arrow pointing upwards on the right-hand side of the diagram indicates the direction of overall volume decrease of the system. The overall Gibbs $(G)$ freeenergy change of folding (4.6 $\mathrm{kcal} / \mathrm{mol}$ ) was determined from $\mathrm{GdnHCl}$-induced denaturation of $\alpha_{3-1}$ (for details, see Ref. 17). The structures shown are artistic views of what the structures of the different conformers stabilized at high pressures and low temperatures may look like. 
states of $\left(\alpha_{2}\right)_{2}$ were stabilized in the unfolding transitions induced by pressure and low temperatures. Interestingly, dimerization of the de novo designed protein accounts for roughly two thirds of the overall free-energy of stabilization determined from $\mathrm{GdnHCl}$ induced equilibrium denaturation of $\left(\alpha_{2}\right)_{2}$, similar to results found for natural protein dimers such as triosephosphate isomerase (21). This means that even such a simplistic de novo designed dimer recapitulates an important feature of natural protein dimers, namely the fact that most of the free energy of stabilization results from intersubunit interactions and not from the independent folding of individual subunits.

\section{Pressure unfolding of natural single- domain proteins}

Pressure denaturation studies of natural single-domain proteins have also revealed the existence of partially folded intermediates. For example, pressure and low temperature denaturation of a tryptophan mutant (F29W) of the $\mathrm{N}$-domain of troponin $\mathrm{C}$, a monomeric protein involved in muscle contraction, stabilized a partially folded conformation that appeared to be similar to that of the $\mathrm{Ca}^{2+}$-bound form (22). High-pressure NMR studies of the globular (87-amino acid residues) Ras-binding domain of the Ral guanine nucleotide dissociation stimulator allowed the stabilization of a partially folded intermediate that exhibited local unfolding in the vicinity of hydrophobic cavities under pressure (23). Pressure-induced and pressure-assisted cold denaturation studies of the natural coiled-coil protein $\alpha$-tropomyosin have also revealed local unfolding and formation of partially folded states in the absence of overall denaturation of the protein $(24,25)$.

\section{Protein misfolding, aggregation and amyloid diseases}

Considerable evidence supports the view that a number of important human pathologies, collectively known as amyloid diseases, share a common etiology related to protein misfolding and aggregation in various organs (26). These pathologies include Alzheimer's, Parkinson's and prion diseases and are related to the conformational conversion of normally innocuous proteins or peptides into toxic species. Even though the underlying molecular mechanisms involved in the formation of toxic protein aggregates are still incompletely understood, a number of studies indicate that stable or metastable alternative protein conformations are linked to the disease-causing misfolding transitions $(27,28)$. Protein aggregates may arise because some of the intermediate structures that are formed during the folding process expose hydrophobic regions on their surfaces, and these regions may bind to similar surfaces in nearby folding molecules instead of becoming buried inside the final folded structure. The problem is made much worse by the high concentrations of macromolecules, including proteins, that are present in cells, but are normally kept under control by proteins called molecular chaperones, which shield the exposed hydrophobic surfaces from one another.

Transmissible spongiform encephalopathies (such as bovine spongiform encephalopathy in cattle and Creutzfeldt-Jakob disease in humans) are associated with the accumulation in the brain of an abnormal protease-resistant form of the prion protein $(\operatorname{PrP})$. Mammalian prion diseases are apparently transmitted directly by PrP and do not involve genetic transmission via nucleic acids (29-31). It is believed that the disease is caused by a conformational change in PrP from a benign cellular conformation $\left(\mathrm{PrP}^{\mathrm{C}}\right)$ to a neurotoxic form $\left(\mathrm{PrP}^{\mathrm{Sc}}\right)$ that self-propagates by recruiting and inducing the conformational change in additional $\mathrm{PrP}^{\mathrm{C}}$ molecules. However, until the present, little is known on the molecular state of the protein that corresponds to the infectious, self-propa- 
gating particle. For example, it is known that one infectious unit corresponds to approximately $10^{5} \mathrm{PrP}$ molecules (32). This could mean that infection is caused by a large aggregate of $\mathrm{PrP}^{\mathrm{Sc}}$ molecules or, alternatively, that a single one of these molecules is actually infectious.

The conformational conversion of the PrP from a predominantly $\alpha$-helical $\left(\operatorname{PrP}^{\mathrm{C}}\right)$ to a $ß$-sheet-rich structure $\left(\mathrm{PrPSc}^{\mathrm{Sc}}\right)$ appears to determine the onset of prion diseases. A number of very recent studies have used hydrostatic pressure to shed light on the folding and aggregation mechanisms of PrP. Highpressure NMR studies of the Syrian hamster $\operatorname{PrP}\left(\mathrm{ShaPrP}_{90-231}\right)$ revealed the existence of a metastable conformer (amounting to only $\sim 1 \%$ of the total protein) that may be related to the transition from $\operatorname{PrP}^{\mathrm{C}}$ to $\operatorname{PrP}^{\mathrm{Sc}}$ (33). A more recent investigation showed that residues localized in regions that are disordered in that metastable conformer exhibit slow conformational fluctuations as measured by NMR (34), suggesting that they may be involved in slow motions of $\mathrm{PrP}^{\mathrm{C}}$ leading to the pathway of formation of $\mathrm{PrPS}^{\mathrm{Sc}}$. The effects of pressure and high temperature on the full-length recombinant murine PrP $\left(\mathrm{mPrP}_{21-231}\right)$ and on an antiparallel $B$-sheetrich isoform ( $(-\mathrm{PrP})$, which shares conformational similarity with $\mathrm{PrP}^{\mathrm{Sc}}$, have recently been studied (35). Those experiments indicate that $\beta-P r P$ is highly sensitive to pressure but resistant to temperature, while the opposite holds for the native protein. These results have been explained by postulating differences in the degree of hydration of the two protein isoforms. Investigation of the unfolding of the structured, C-terminal domain of recombinant $\mathrm{mPrP} \mathrm{P}_{121-231}$ using high pressures and low temperatures pointed to the existence of two partially folded states of $\mathrm{PrP}$ which might be involved in the $\mathrm{PrPC}^{\mathrm{C}} \rightarrow$ $\mathrm{PrP}^{\mathrm{Sc}}$ conversion or in the formation of neurotoxic PrP species in vivo (36). Pressureinduced unfolding of the $\mathrm{ShaPrP}_{90-231}$ has also been reported (37). Interestingly, that study showed thioflavin- $\mathrm{T}$ binding to the PrP above $400 \mathrm{MPa}$, suggesting the formation of prion amyloid aggregates at elevated pressure. A subsequent study showed that pressure converts the recombinant hamster PrP into a misfolded conformation which is prone to aggregation and forms amyloid fibrils (38). The sensitivity of recombinant prion aggregates to pressure has also been investigated (39). Of significant biotechnological interest, the pressure-sensitivity of prion aggregates has recently been explored as a tool to inactivate scrapie infectivity in brain homogenates and in processed meat derivatives $(40,41)$.

Another interesting recent application of hydrostatic pressure has been in the investigation of the misfolding and amyloid aggregation of transthyretin, which is involved in human senile systemic amyloidosis and in amyloidotic familiar polyneuropathy $(1,42-$ $45)$. The interested reader is referred to a recent review on this topic (46). Below, we present a brief discussion of recent developments in the use of pressure to investigate amyloid formation from human lysozyme and its relationship with systemic amyloidosis.

Human lysozyme is involved in the formation of amyloid deposits in autosomal hereditary systemic amyloidosis (47). This disease is associated with point mutations in the lysozyme gene, causing the nonconservative substitutions Ile56Thr or Asp67His, and amyloid fibrils are widely deposited in a variety of tissues (48). The propensities of lysozyme variants to form amyloid fibrils appear to be due to their reduced thermodynamic stabilities and enhanced abilities to form partially unfolded amyloidogenic states $(49,50)$. It has also been shown that wildtype (WT) lysozyme forms amyloid fibrils in vitro upon prolonged incubation at $\mathrm{pH} 2.0$ and elevated temperatures (51). Consistent with their reduced thermodynamic stabilities, Ile56Thr and Asp67His lysozyme variants form amyloid fibrils upon incubation at 
lower temperatures than are required for aggregation of the WT protein $(49,51)$.

In a recent study, hydrostatic pressure has been used as a tool to generate the amyloidogenic conformation of lysozyme without the need to introduce modifications in the chemical composition of the medium (i.e., amyloid aggregation was achieved at physiological $\mathrm{pH}$ in the absence of chemical denaturants) (52). Interestingly, both WT lysozyme and its variants remain soluble upon incubation at high pressure, and aggregation to amyloid structures takes place only upon decompression of the samples. This suggests that application of pressure leads to conversion of lysozyme from its native conformation to a potentially amyloidogenic state which, however, does not form aggregates while the pressure is maintained.

It is well known that high pressures promote subunit dissociation, or impede the association, of oligomeric proteins (for reviews, see Refs. 53,54). This effect seems to be primarily due to pressure-induced destabilization of hydrophobic interactions that are important for the assembly of protein oligomers and that have been shown to play important roles in the stability of amyloid structures (for recent examples, see Refs. 55-57). Thus, it seems likely that pressure perturbs hydrophobic interactions that are important for the stability of lysozyme aggregates and prevents any amyloidogenic intermediates formed in solution from un- dergoing self-association to form high-molecular weight assemblies. Spectroscopic measurements carried out under pressure suggest that compression causes only subtle conformational changes in lysozyme. Nevertheless, the changes appear to be sufficient to cause aggregation and amyloid formation upon decompression (52). Regarding the mechanism of pressure-induced amyloid formation, one possibility is that a partially unfolded state of lysozyme populated under pressure forms oligomeric nuclei that act as seeds for further aggregation upon decompression. Alternatively, decompression may trigger a kinetic partitioning between refolding of lysozyme and its interaction with other partially unfolded molecules, leading to aggregation. Regardless of the exact mechanism involved, it is remarkable that globular aggregates, protofibrils and mature amyloid fibrils can be formed by WT and mutant lysozymes after a pressure cycle.

Hydrostatic pressure perturbation may represent an important tool to gain insight into the mechanisms and pathways of productive protein folding as well as to characterize the formation of partially folded, amyloidogenic states of proteins. In addition to shedding light on an intriguing and still relatively obscure biological problem, a deeper understanding of the initial stages of protein aggregation may also assist in drug design approaches that target inhibitors of amyloidogenesis.

\section{References}

1. Kelly JW (1998). The alternative conformations of amyloidogenic proteins and their multi-step assembly pathways. Current Opinion in Structural Biology, 8: 101-106.

2. Dobson CM (2004). Experimental investigation of protein folding and misfolding. Methods, 34: 4-14.

3. Degrado WF, Summa CM, Pavone V et al. (1999). De novo design and structural characterization of proteins and metalloproteins. Annual Review of Biochemistry, 68: 779-819.

4. Hill CP, Anderson DH, Wesson L et al. (1990). Crystal structure of alpha 1: implications for protein design. Science, 249: 543-546.
5. Harbury PB, Zhang T, Kim PS et al. (1993). A switch between two-, three-, and four-stranded coiled coils in GCN4 leucine zipper mutants. Science, 262: 1401-1407.

6. Zhou $Y$ \& Karplus M (1999). Interpreting the folding kinetics of helical proteins. Nature, 401: 400-403.

7. Zipp A \& Kauzmann W (1973). Pressure denaturation of metmyoglobin. Biochemistry, 12: 4217-4228.

8. Edelhoch H \& Osborne Jr JC (1976). The thermodynamic basis of the stability of proteins, nucleic acids, and membranes. Advances in Protein Chemistry, 30: 183-250. 
9. Honig BH, Hubbel WL \& Flewelling RF (1986). Electrostatic interactions in membranes and proteins. Annual Review of Biophysics and Biophysical Chemistry, 15: 163-193.

10. Masterton WL (1954). Partial molar volumes of hydrocarbons in solution. Journal of Chemical Physics, 22: 1830-1833.

11. Masterton WL \& Seiler H (1968). Apparent and partial molar volumes of water in organic solvents. Journal of Chemical Physics, 72: 4257-4262.

12. Frye KJ \& Royer CA (1998). Probing the contribution of internal cavities to the volume change of protein unfolding under pressure. Protein Science, 7: 2217-2222.

13. Lopes DH, Chapeaurouge A, Manderson GA et al. (2004). Redesigning the folding energetics of a model three-helix bundle protein by site-directed mutagenesis. Journal of Biological Chemistry, 279: 10991-10996.

14. Silva JL, Foguel D \& Royer CA (2001). Pressure provides new insights into protein folding, dynamics and structure. Trends in Biochemical Sciences, 26: 612-618.

15. Foguel D \& Silva JL (2004). New insights into the mechanisms of protein misfolding and aggregation in amyloidogenic diseases derived from pressure studies. Biochemistry, 43: 11361-11370.

16. Johansson JS, Gibney BR, Skalicky JJ et al. (1998). A native-like three-helix bundle protein from structure-based redesign: a novel maquette scaffold. Journal of the American Chemical Society, 120: 3881-3886.

17. Chapeaurouge A, Johansson JS \& Ferreira ST (2001). Folding intermediates of a model three-helix bundle protein. Pressure and cold denaturation studies. Journal of Biological Chemistry, 276: 14861-14866.

18. Bridgman PW (1931). The Physics of High Pressure. G. Bell and Sons Ltd., London, UK.

19. Privalov PL (1990). Cold denaturation of proteins. Critical Reviews in Biochemistry and Molecular Biology, 25: 281-305.

20. Chapeaurouge A, Johansson JS \& Ferreira ST (2002). Folding of a de novo designed native-like four-helix bundle protein. Journal of Biological Chemistry, 277: 16478-16483.

21. Rietveld AW \& Ferreira ST (1998). Kinetics and energetics of subunit dissociation/unfolding of TIM: the importance of oligomerization for conformational persistence and chemical stability of proteins. Biochemistry, 37: 933-937.

22. Foguel D, Suarez MC, Barbosa C et al. (1996). Mimicry of the calcium-induced conformational state of troponin $\mathrm{C}$ by low temperature under pressure. Proceedings of the National Academy of Sciences, USA, 93: 10642-10646.

23. Inoue K, Yamada H, Akasaka K et al. (2000). Pressure-induced local unfolding of the Ras binding domain of RalGDS. Nature Structural Biology, 7: 547-550.

24. Suarez MC, Lehrer SS \& Silva JL (2001). Local heterogeneity in the pressure denaturation of the coiled-coil tropomyosin because of subdomain folding units. Biochemistry, 40: 1300-1307.

25. Ferreira ST \& Morais AC (2005). Folding and stability of a coiled-coil investigated using chemical and physical denaturing agents: Comparative analysis of polymerized and non-polymerized forms of alpha-tropomyosin. International Journal of Biochemistry and Cell Biology, 37: 1386-1395.

26. Soto $C$ (2003). Unfolding the role of protein misfolding in neurodegenerative diseases. Nature Reviews. Neuroscience, 4: 49-60.

27. Carrell RW \& Lomas DA (1997). Conformational disease. Lancet, 350: 134-138.

28. Soto $C$ (2001). Protein misfolding and disease; protein refolding and therapy. FEBS Letters, 498: 204-207.
29. Gajdusek DC, Gibbs CJ \& Alpers M (1966). Experimental transmission of a Kuru-like syndrome to chimpanzees. Nature, 209: 794-796.

30. Gajdusek DC, Gibbs Jr CJ \& Alpers M (1967). Transmission and passage of experimental "Kuru" to chimpanzees. Science, 155: 212214.

31. Prusiner SB (1998). Prions. Proceedings of the National Academy of Sciences, USA, 95: 13363-13383.

32. Bolton DC, McKinley MP \& Prusiner SB (1982). Identification of a protein that purifies with the scrapie prion. Science, 218: 1309-1311.

33. Kuwata K, Li H, Yamada $\mathrm{H}$ et al. (2002). Locally disordered conformer of the hamster prion protein: a crucial intermediate to PRPSC? Biochemistry, 41: 12277-12283.

34. Kuwata K, Kamatari YO, Akasaka K et al. (2004). Slow conformational dynamics in the hamster prion protein. Biochemistry, 43: 4439-4446.

35. Cordeiro Y, Kraineva J, Ravindra R et al. (2004). Hydration and packing effects on prion folding and beta-sheet conversion. High pressure spectroscopy and pressure perturbation calorimetry studies. Journal of Biological Chemistry, 279: 32354-32359.

36. Martins SM, Chapeaurouge A \& Ferreira ST (2003). Folding intermediates of the prion protein stabilized by hydrostatic pressure and low temperature. Journal of Biological Chemistry, 278: 50449-50455.

37. Torrent J, Alvarez-Martinez MT, Heitz F et al. (2003). Alternative prion structural changes revealed by high pressure. Biochemistry, 42: 1318-1325.

38. Torrent J, Alvarez-Martinez MT, Harricane MC et al. (2004). High pressure induces scrapie-like prion protein misfolding and amyloid fibril formation. Biochemistry, 43: 7162-7170.

39. Alvarez-Martinez MT, Torrent J, Lange R et al. (2003). Optimized overproduction, purification, characterization and high-pressure sensitivity of the prion protein in the native $(\operatorname{prp}(\mathrm{c})$-like) or amyloid (PRP(SC)-like) conformation. Biochimica et Biophysica Acta, 1645: 228-240.

40. Brown P, Meyer R, Cardone F et al. (2003). Ultra-high-pressure inactivation of prion infectivity in processed meat: a practical method to prevent human infection. Proceedings of the National Academy of Sciences, USA, 100: 6093-6097.

41. Garcia AF, Heindl $P$, Voigt $H$ et al. (2004). A dual nature of the infectious prion protein revealed by high pressure. Journal of Biological Chemistry, 280: 9842-9847.

42. Kelly JW, Colon W, Lai Z et al. (1997). Transthyretin quaternary and tertiary structural changes facilitate misassembly into amyloid. $A d$ vances in Protein Chemistry, 50: 161-181.

43. Saraiva MJ (2003). Cellular consequences of transthyretin deposition. Amyloid, 10 (Suppl 1): 13-16.

44. Sousa MM \& Saraiva MJ (2003). Neurodegeneration in familial amyloid polyneuropathy: from pathology to molecular signaling. Progress in Neurobiology, 71: 385-400.

45. Hurshman AR, White JT, Powers ET et al. (2004). Transthyretin aggregation under partially denaturing conditions is a downhill polymerization. Biochemistry, 43: 7365-7381.

46. Foguel D (2005). High pressure studies on transthyretin. Protein and Peptide Letters, 12: 245-249.

47. Pepys MB, Hawkins PN, Booth DR et al. (1993). Human lysozyme gene mutations cause hereditary systemic amyloidosis. Nature, 362: 553-557.

48. Booth DR, Sunde M, Bellotti V et al. (1997). Instability, unfolding and aggregation of human lysozyme variants underlying amyloid fibrillogenesis. Nature, 385: 787-793.

49. Canet D, Sunde M, Last AM et al. (1999). Mechanistic studies of the folding of human lysozyme and the origin of amyloidogenic behavior 
in its disease-related variants. Biochemistry, 38: 6419-6427.

50. Canet D, Last AM, Tito P et al. (2002). Local cooperativity in the unfolding of an amyloidogenic variant of human lysozyme. Nature Structural Biology, 9: 308-315.

51. Morozova-Roche LA, Zurdo J, Spencer A et al. (2000). Amyloid fibril formation and seeding by wild-type human lysozyme and its disease-related mutational variants. Journal of Structural Biology, 130: 339-351.

52. De Felice FG, Vieira MN, Meirelles MN et al. (2004). Formation of amyloid aggregates from human lysozyme and its disease-associated variants using hydrostatic pressure. FASEB Journal, 18: 10991101.

53. Gross M \& Jaenicke R (1994). Proteins under pressure. the influence of high hydrostatic pressure on structure, function and assembly of proteins and protein complexes. European Journal of Bio- chemistry, 221: 617-630.

54. Mozhaev VV, Heremans K, Frank J et al. (1996). High pressure effects on protein structure and function. Proteins, 24: 81-91.

55. De Felice FG, Houzel JC, Garcia-Abreu J et al. (2001). Inhibition of Alzheimer's disease beta-amyloid aggregation, neurotoxicity, and in vivo deposition by nitrophenols: implications for Alzheimer's therapy. FASEB Journal, 15: 1297-1299.

56. Chiti F, Taddei N, Baroni F et al. (2002). Kinetic partitioning of protein folding and aggregation. Nature Structural Biology, 9: 137143.

57. Kapurniotu A, Schmauder A \& Tenidis K (2002). Structure-based design and study of non-amyloidogenic, double n-methylated IAPP amyloid core sequences as inhibitors of IAPP amyloid formation and cytotoxicity. Journal of Molecular Biology, 315: 339-350. 